\title{
An intelligent hospital operating room to improve patient health care
}

Marc Garbey ${ }^{1,3,4}$, Guillaume Joerger ${ }^{2}$, Albert Huang ${ }^{3 *}$, Remi Salmon ${ }^{4}$, Jinsu Kim5 ${ }^{5}$ Vadim Sherman ${ }^{3}$, Brian Dunkin ${ }^{3}$ and Barbara Bass ${ }^{3}$

\author{
* Correspondence: \\ ayhuang@houstonmethodist.org \\ ${ }^{3}$ Department of Surgery, Houston \\ Methodist Hospital, 6550 Fannin St. \\ Suite 1661, Houston TX 77030, USA \\ Full list of author information is \\ available at the end of the article
}

\begin{abstract}
Optimizing management of multiple hospital operating rooms (ORs) is a complex problem. A large hospital can have upwards of greater than 50 with a large number of different procedures per day and per OR that needs to be scheduled several weeks in advance. Each procedure requires gathering a team led by a surgeon for a specific block of time in the OR, but even common procedures such as cholecystectomies, which account for about 1.4 million cases per year in the USA, exhibit a significant variation in total procedure duration. OR time is one of the most significant budget chapters in a modern hospital, and it has also been shown that delays in OR procedures due to lapses in scheduling and/or OR resources availability have been responsible for post-surgical complications. We propose an innovative, cost effective hardware/software OR awareness solution that automatically (i) detects what step of the procedure the OR team is at, (ii) determines if steps are out of order, (iii) identifies and pinpoints procedural delays, irregularities, and unused OR time, and (iv) can assist in root cause analyses and assessment. Most institutions have an electronic OR management software system in place that allows for easy projection and visualization of the daily OR schedule, but all rely heavily on manual data entry resulting in human error and bias. There exists a need not only for a fully automated, unbiased, and accurate OR management system that also collects key procedural data for both real-time and retrospective analyses but also for an intuitive and user-friendly digital interface. With our system, we have been able to track and collect data on almost 300 cases to not only identify but quantify sources of inefficiency as well as automatically indicate cases that exceed expected lengths.
\end{abstract}

Keywords: Safety; OR efficiency; OR management; Outcomes; Surgical; Technology

\section{Background}

We started the smart operating room (OR) concept to aid surgeons, OR staff, as well as patients and patient family members that have expressed their frustration in excessive waiting times as well as delays in and out of the OR. Difficulties in efficient communication between OR staff and those team members responsible for organizing and managing the OR suite activities also exists in every hospital across the nation [1]. An OR suite at a hospital is a system constantly in flux. Cases are added and canceled regularly, rooms need to be reallocated, and a significant amount of coordination is required to know current 
room statuses for each OR in order to guide staff and patient movement in the perioperative space.

The hospital management is constantly struggling to improve the efficiency of OR usage, as it is becoming a major issue to provide high quality affordable health care. At a cost of approximately $\$ 100$ per min, OR time is incredibly expensive and even small delays compounded over the course of multiple ORs and multiple days result in a significant impact [2].

We have assembled an interdisciplinary team of surgeons, applied mathematicians, and computer scientists to focus on two key aspects and needs to improve OR efficiency:

1. How do we close the information loop between the surgical team and the OR system, so the OR system can add computational intelligence to deliver the procedures with allocation of OR time and resources in an optimal way?

2. Can we provide context information on patient status from patient registration to patient discharge, since it is well known that inefficiency and safety issues may come from miscommunications with the OR team at both the preparation and recovery stage?

The different backgrounds (from health care to design) of the team allow us to approach each problem from multiple perspectives as well as maintaining a translational dialog in the hospital. Not only combining engineers, programmers, and user interface designers but also having dedicated surgeons on the team ensures that the project design can be smoothly implemented while remaining cognizant of the complex functions and the "culture" of the operating room and perioperative space. Just as importantly, we have nurse managers and OR staff members as regular consultants that can describe the issues they encounter on a daily basis in the perioperative space.

The missing link in today's hospital system is that there is no rigorous way of automatically collecting information on what is occurring in the OR and the perioperative space [3]. Most current systems are error prone because they require a fairly large amount of tedious human work and intervention while health care personnel are usually already very busy with their duties [4]. Some systems rely completely on radio frequency identification (RFID) as well which is expensive and requires complex installation and tag deployment [5]. We endeavor to propose a modern solution that would work for any operation but would be most efficient for large volume procedures. The major steps of an OR cycle (Fig. 1) from the patient entering the OR through the cleaning after a case is completed can be segmented, with at least one specific event occurring at each step that can be targeted with a simple, dedicated sensor.

For technology acceptance, it is essential to have sensors that target tasks but not necessarily specific staff members. The system of distributed sensors also needs to require as little maintenance as possible, requires no sterilization, and most importantly, does change the overall flow of the OR.

\section{Methods}

For our project, we first segmented the operating room cycle into sequential events and then identified an action that was unique to each event. We then developed a dedicated sensor that would be designed to capture the occurrence of that event (Table 1). The first 


\section{Room States

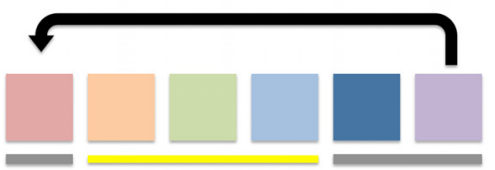 \\ Clean Room/Unoccupied \\ Intubation/Start of Procedure \\ Laparoscopic Portion of Procedure \\ End of Procedure/Extubation \\ Dirty Room \\ Cleaning/Set Up \\ Total Procedure Time \\ Turnover Time}

Fig. 1 Operating room states and cycle. Representation of the OR cycle

goal was to ensure that the system was robust enough to build a timeline of events during a day in the operating room and to be able to collect and transmit these data wirelessly to a centralized computer for consolidation and analyses.

Each designed sensor was built with custom communication software protocols as well as being wifi-enabled to allow for wireless connectivity and ease of installation. Three sensors were placed in each OR in key locations to capture target events while remaining out of the way and unobtrusive to the OR team. For this study, two ORs adjacent to each other were outfitted with the three sensor array (Fig. 2) and both communicated via a secure wireless network to the central computer that not only projected the sensor outputs in real-time but also captured the data for retrospective analyses. The system was installed and running $24 \mathrm{~h}$ a day for 7 days a week beginning at the end of 2014.

Table 1 Sensors targeting key events at each step of the procedure

\begin{tabular}{|c|c|c|}
\hline Step of procedure & Event target & Sensor type \\
\hline Instrument/back table setup & $\begin{array}{l}\text { Instrument racks placed } \\
\text { on table }\end{array}$ & Pressure sensing strip \\
\hline Patient brought into OR & Stretcher crossing door threshold & Pressure sensing strip \\
\hline Patient transferred to OR table & $\begin{array}{l}\text { Patient weight transferred from } \\
\text { stretcher to table }\end{array}$ & Pressure sensing strip \\
\hline Induction of anesthesia & Ventilation initiation & Small motion detection camera \\
\hline Operation & First incision & Scalpel pick up detection \\
\hline Reversal of anesthesia/extubate & Ventilator cessation & $\begin{array}{l}\text { Small motion detection camera } \\
\text { (same one as induction of anesthesia) }\end{array}$ \\
\hline Patient transferred to stretcher & $\begin{array}{l}\text { Patient weight transferred from table } \\
\text { to stretcher }\end{array}$ & $\begin{array}{l}\text { Pressure sensing strip } \\
\text { (same one as prior) }\end{array}$ \\
\hline Patient taken to recovery room & Stretcher crossing door threshold & $\begin{array}{l}\text { Pressure sensing strip } \\
\text { (same one as prior) }\end{array}$ \\
\hline Occupancy of the OR & Movement in the $\mathrm{OR}$ & Infrared detector \\
\hline
\end{tabular}




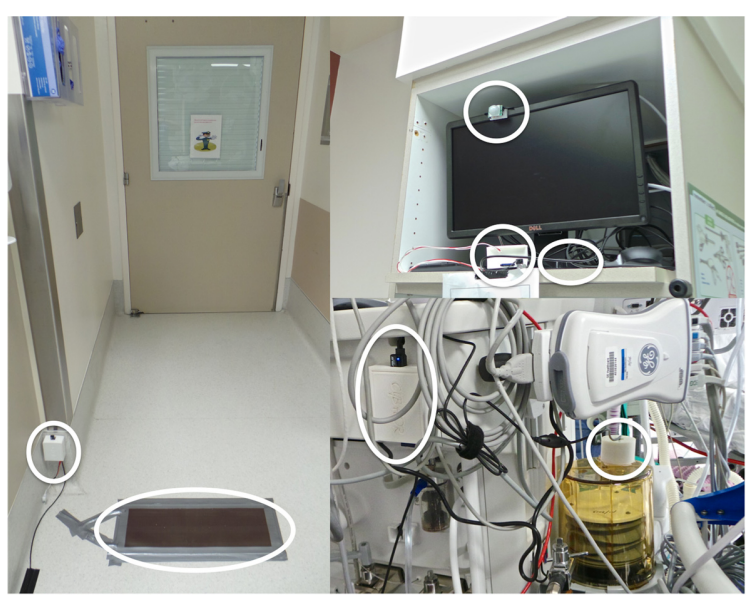

Fig. 2 Installation of the smart OR system. Central computer where the data is collected from the sensors via 4G server (top right). IR sensor is also placed on the computer. Other images show the sensors installed in the OR. A camera in a 3D printed box (bottom right) is placed on the top of the ventilator bellows. Finally, two parallel pressure sensors protected by a plastic mattress placed at the doorstep (left)

To focus on potential events leading to delays of when the first surgical case starts (ultimately resulting in delays through the rest of the day), we installed a dedicated RFID system in the preoperative space where surgeons must see their patient before the patient can be taken to the OR (Fig. 3). Three staff surgeons were provided unique RFID tags to capture the time(s) that they entered the preoperative area. The goal was to first see how soon before the scheduled start time for the first case of the day they visited their patient and how often the first case starts on time.

All the sensors were remotely controllable for fast and noninvasive potential system repairs. All components were easily acquired and assembled with 3D printed cases. Only a small white box and the sensor or camera are visible in the installed system, and all sensors were plugged into the emergency power outlets in and around the OR as these had dedicated uninterrupted power. The collected data were always anonymous as they centered around case types and perioperative setting movements and functions and not on individuals. The data stream was stored on an on-site secure

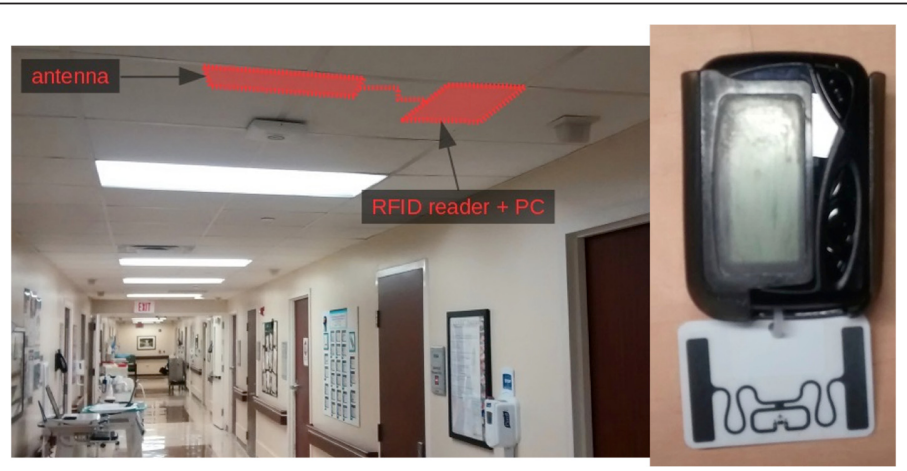

Fig. 3 RFID system installed in the preoperative area. The system is placed above ceiling and unique tags are worn by staff surgeons 
server, and prior to installation, all our sensors and common wireless communication methods were evaluated and cleared by the hospital administration and the information technology department.

\section{Results and discussion}

With the collection and processing of 281 cases collected from 12/1/14 to 5/1/14, results obtained confirm the capability of accurately identifying the steps of the operative procedure outlined in Table 1 in a repeatable and reliable way. A representative generated timeline of sensor-derived data from a single OR on 1 day is depicted in Fig. 4. The doorway pressure sensor reliably detected entrance and exit of the patient bed and initiation as well as cessation of the ventilator function (indicating when the patient was intubated as well as extubated) was clearly captured by the motion sensor camera placed on the ventilator bellows. On the generated timeline, changes in ambient light within the OR are visually depicted as a decrease in peak intensity of the ventilator movement and signify "going green" in the room for the laparoscopic portion of the operation.

The database we built gives us access to a very broad range of types of procedures from a range of surgical specialties. While performing the data analyses and segmentation, we did statistical analyses on each part of the OR cycle. We also separated out the different types of procedures and did a more in depth analysis of common general

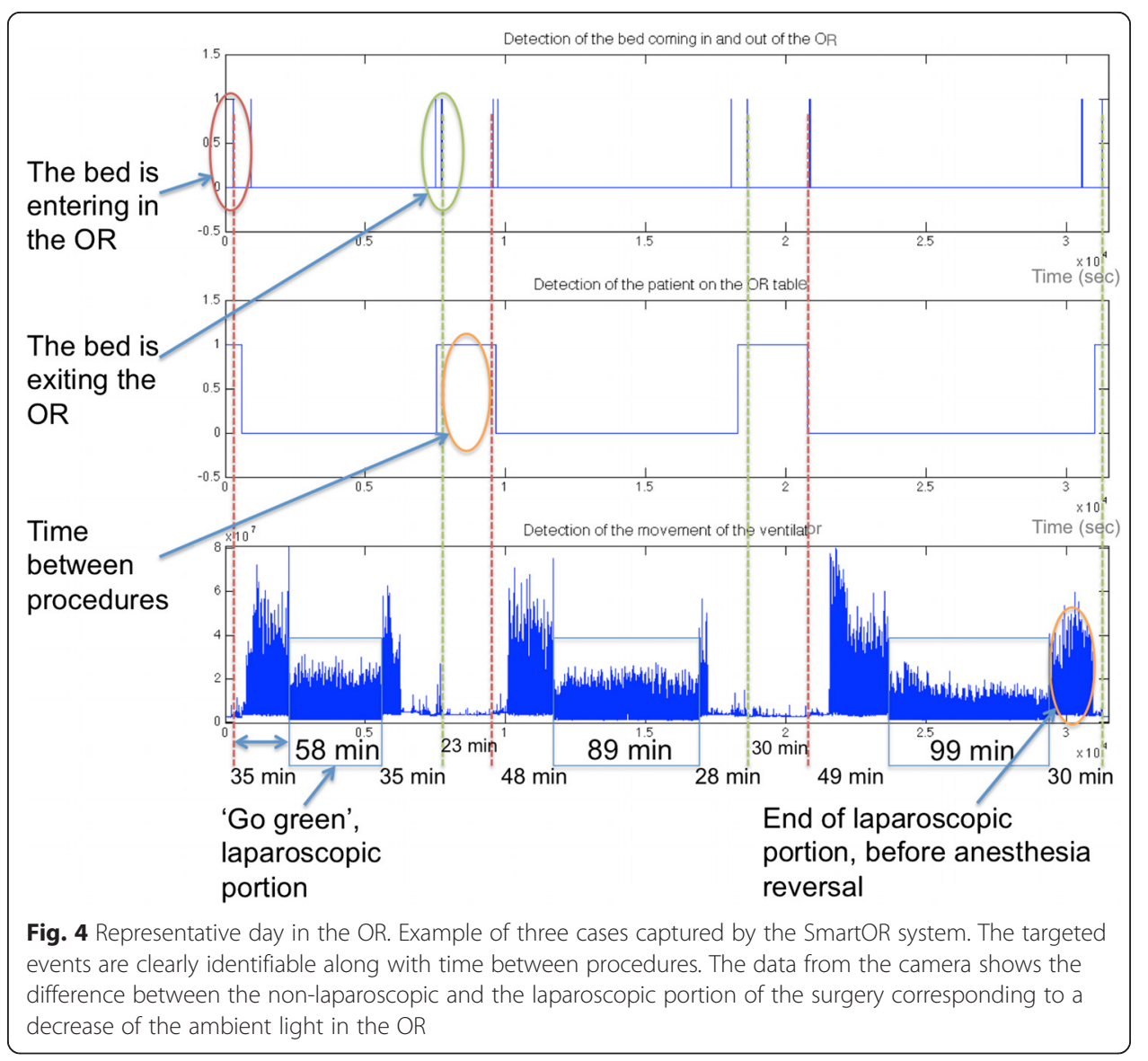


surgery procedures. For clinical relevance, we focused on examining the outliers in each portion of the OR cycle and overall outliers in common general surgery procedures.

A case is defined as an outlier as soon as its length in, at least, one part of the operation extends the mean plus 1 standard deviation from the other cases. With outliers identified, we can track back the reason(s) of the delay(s) during (or before/ after) the operation on the electronic medical record (EMR). This way, the feedback we can give to the OR staff is stronger because it has this post processing value that no other system could have before. For example, our system captured an operation in which the patient spent a significantly extended length of time in the OR (Fig. 5a) in comparison to over the 256 cases analyzed (open or laparoscopic ones but with intubation). This case exceeded the rest of the cases by the mean (174 min) plus 6 standard deviations ( $1 \mathrm{SD}=83 \mathrm{~min}$ ). Going back to the EMR, we understood that this case was a very complex one with different surgeons involved.

To focus on portions of the OR cycle that should have an approximate average time frame irrespective of the type of procedure performed (and which could be a realistic focal point for OR efficiency improvement), we focused then on the time between when

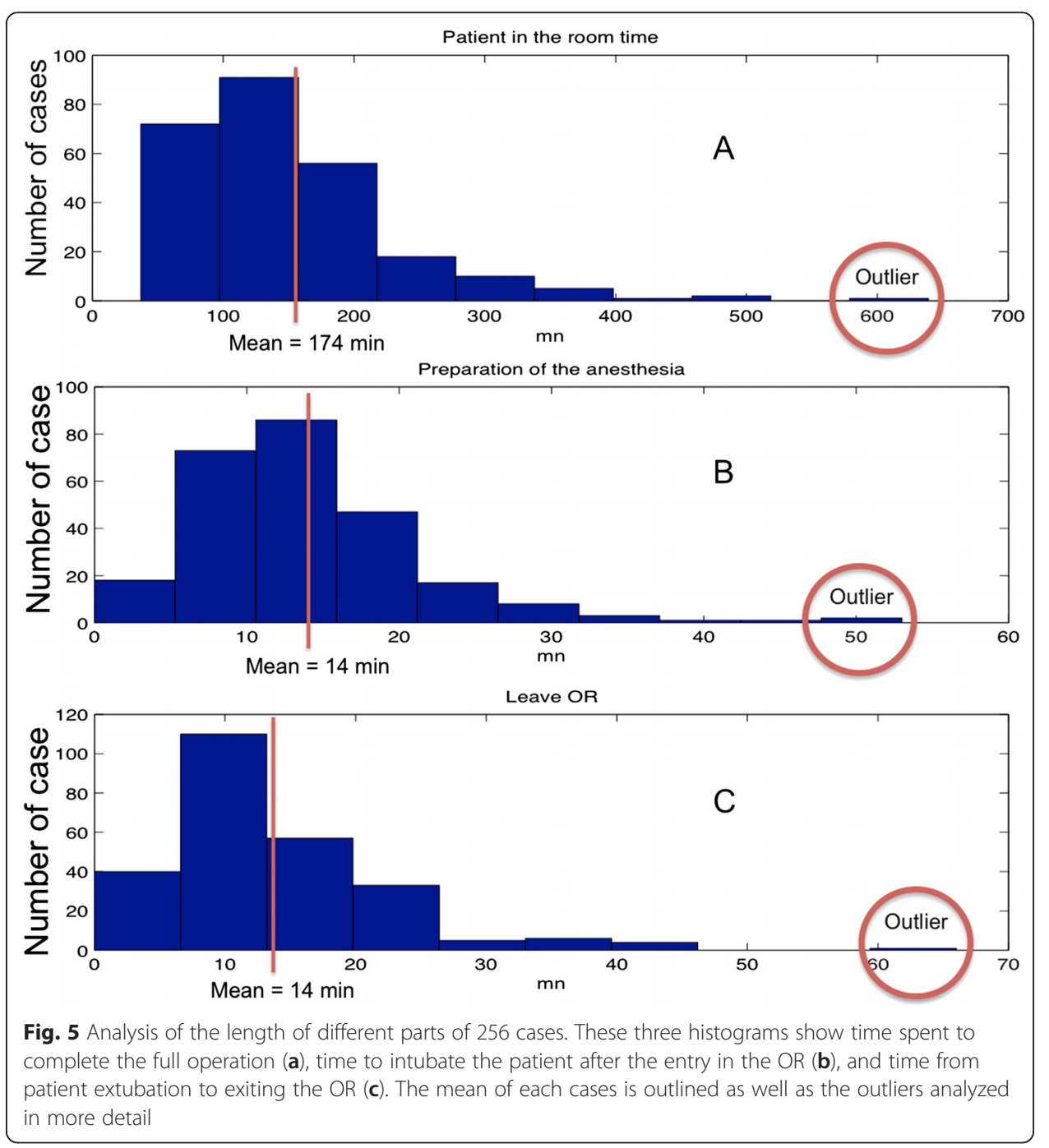


the patient enter (bed detected) and the ventilation starts (the bellows starts to move) as well as the time between the end of the ventilation and the patient exiting the room. As before, we analyzed 256 cases, computed the mean and standard deviations, then identified outliers. We found that overall, $23 \%$ of the cases were suboptimal in one of this two parts. Here as well, some cases had longer durations in these portions of the operation than other. If we take the example of the case circled on Fig. 5b which exceeded 6 standard deviations $(1 \mathrm{SD}=8 \mathrm{~min}$ ), this indicated that there were significant and unusual circumstances that prevented the procedure from progressing in an expected fashion. When we referred back to the anesthesiologist's record in the EMR, we were able to see segments of time between when the patient entered the room and when they were intubated where no activity was noted even though this time frame was nearly $1 \mathrm{~h}$ in length.

On the other end of the operation, between when the patient was extubated and exited the room, the system identified cases that again exceeded the mean by 6 standard deviations (Fig. 5c). Upon reviewing the case details, there were multiple etiologists to these delays with the most common being that the recovery room was not yet ready to receive the patient.

With or without reported causes in the EMR, the system has proven its ability and usefulness in better understanding and identifying problem points of OR workflow on a broad range and number of cases. The system can be leveraged to focus on very specific case types as well. When filtering for laparoscopic cholecystectomies (one of the most common general surgery procedures), our system had captured and analyzed ten of them and identified one large outlier (Fig. 6) in overall case length that was reported on the EMR as a delay due to the recovery room not being ready to accept the patient from the OR.

When focus is turned to the non-procedural portions of the OR cycle such as the time between cases (turnover time), the system demonstrates how easily and precisely it can automatically detect, quantify, and generate meaningful data to be used by the team to improve efficiency. At our institution, the ideal turnover time is $30 \mathrm{~min}$. With the system tracking cases, we determined the mean time to be $35 \mathrm{~min}$ (Fig. 7) indicating an opportunity to improve efficiency.

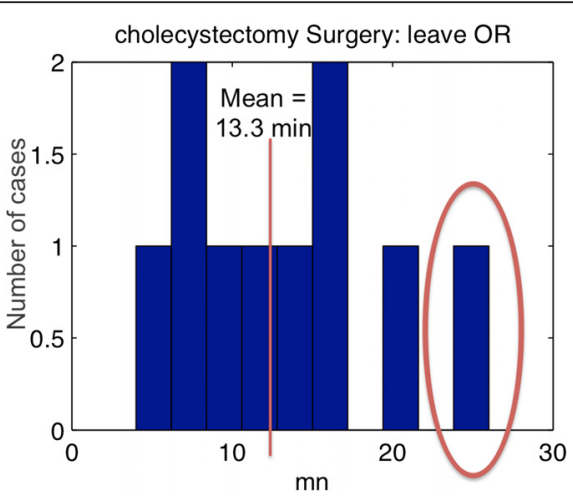

Fig. 6 Distribution of time spent between extubation and the patient leaving the OR. We analyzed ten laparoscopic cholecystectomies, and here, we focus on the time after extubation and when the patient exits the OR. The system determines the mean and identifies clear outliers 


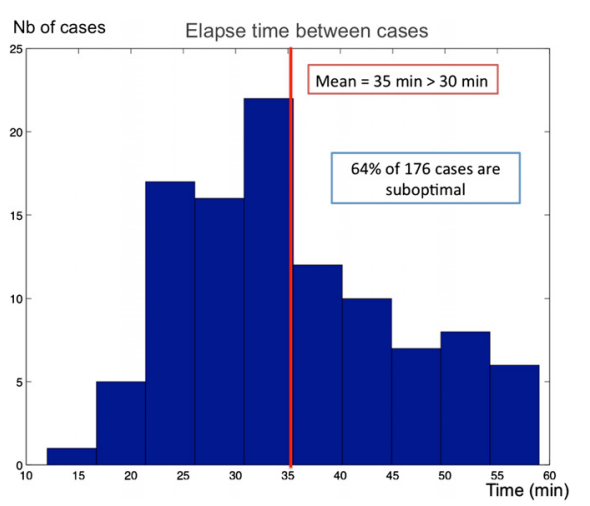

Fig. 7 Distribution of the elapsed time between cases. The analysis of turnover time. To ensure accuracy, we eliminated times greater than 60 min to exclude nighttime and singular operations

Finally, with the RFID component of our system, we were able to track when the three surgeons identified their first patient of the day, and then with our OR, sensors determine the actual time the patient entered the OR versus the scheduled time. We found that the average time the surgeons visited the patient was approximately $8 \mathrm{~min}$ prior to the time the case was schedule to start and that $69 \%$ of the first cases of the day started late (Table 2). The implications here are large as a late start to the first case of the day in an OR can easily result in delays over the course of the day in that OR.

Table 2 Correlation of first case start times and output from RFID system in the preoperative area

\begin{tabular}{llllll}
\hline Date & RFID time & $\begin{array}{c}\text { Scheduled OR time } \\
\text { (first case start) }\end{array}$ & $\begin{array}{l}\text { Actual patient } \\
\text { in-room time }\end{array}$ & $\begin{array}{l}\text { Documented } \\
\text { delay cause }\end{array}$ & $\begin{array}{l}\text { Minutes between } \\
\text { RFID and } \\
\text { in-room time }\end{array}$ \\
\hline 03/19/15 & $7: 23: 17$ & $7: 30$ & $7: 30$ & N/A & $0: 06: 43$ \\
03/23/15 & $7: 27: 34$ & $7: 30$ & $7: 40$ & Staff surgeon related & $0: 12: 26$ \\
03/23/15 & $7: 10: 01$ & $7: 30$ & $7: 30$ & N/A & 0:19:59 \\
03/24/15 & $7: 20: 20$ & $7: 30$ & $7: 29$ & N/A & $0: 08: 40$ \\
03/26/15 & $7: 08: 43$ & $7: 30$ & $7: 29$ & N/A & $0: 20: 17$ \\
03/30/15 & $7: 31: 19$ & $7: 30$ & $7: 52$ & Staff surgeon related & $0: 20: 41$ \\
04/01/15 & $8: 57: 08$ & $9: 00$ & $9: 02$ & Staff surgeon related & $0: 04: 52$ \\
04/02/15 & $7: 16: 48$ & $7: 30$ & $7: 35$ & Staff surgeon related & $0: 18: 12$ \\
04/06/15 & $7: 31: 37$ & $7: 30$ & $7: 38$ & N/A & $0: 06: 23$ \\
04/07/15 & $7: 32: 15$ & $7: 30$ & $7: 42$ & Staff surgeon related & $0: 09: 45$ \\
04/08/15 & $7: 49: 50$ & $9: 00$ & $9: 14$ & Staff nursing related & $1: 24: 10$ \\
04/10/15 & $7: 26: 35$ & $7: 30$ & $7: 34$ & Staff surgeon related & $0: 07: 25$ \\
04/16/15 & $7: 24: 07$ & $7: 30$ & $7: 30$ & N/A & $0: 05: 53$ \\
04/20/15 & $7: 38: 03$ & $7: 30$ & $7: 41$ & Staff surgeon related & $0: 02: 57$ \\
04/20/15 & $7: 28: 45$ & $7: 30$ & $7: 39$ & Staff surgeon related & $0: 10: 15$ \\
04/27/15 & $6: 47: 41$ & $7: 30$ & $7: 36$ & Staff surgeon related & $0: 48: 19$ \\
Mean & $7: 21: 56$ & & $69 \%$ late & & $0: 17: 56$ \\
(for 7:30 & & & & & Mean \\
start times) & & & & & \\
\hline
\end{tabular}




\section{Conclusions}

With this ongoing study, we have demonstrated that by using a refined set of simple, wireless sensors, we can accurately and effectively capture key events in the cycle of the operating room. Retrospective analyses of these collected data also allow us to quickly and easily generate clinically and operationally relevant statistical measurements that can be used as focal points for OR efficiency improvements. As the data collection continues to increase, ultimately, correlations between events and event lengths with patient outcomes may become possible. This would fully leverage the system's ability to automatically track and record OR activity and result in a refined system that can notify appropriate OR staff/ team members if certain events or event lengths are risking patient safety in real-time. Several intraoperative events such as length of time in the OR as well as time under anesthesia contribute to poor patient outcomes, and our system can assist in preventing these [6].

The final goal of the system is to build a new, efficient, dynamic, and real-time OR management platform. Along with hardware and software refinement, we also plan to add the capability of patient localization. To address this, we are currently testing small programmable radio frequency modules that can be either worn by the patient or attached to the patient's chart as it travels with the patient in the perioperative space as well as when they are transported for procedures or imaging around the hospital.

The user interface of the system will be of high importance as well. Building an intuitive graphic user interface that distils the information collected in real-time by the sensor network will bring the system together in a centralized, interactive screen. This will provide real-time updates, patient locations, as well as the status of each OR with the touch of a button. Our system will also be able to interface with EMRs via the now-standardized HL7 (health level 7) protocols for patient data transmission. Ultimately, this interface will be the product that the end user interacts with. All the data collection, patient and procedural tracking, as well as statistical analyses and implementation will all be done behind that interface.

Abbreviations

EMR: electronic medical record; HL7: health level 7; OR: operating room; RFID: radio frequency identification; SD: standard deviation.

Competing interests

The authors declare that they have no competing interests.

Authors' contributions

GJ, AH, and RS designed and constructed the hardware for the system along with deployment of the system in the perioperative space. Data processing and automation was also done by GJ, AH, and RS. JK designed and programmed the software. BB, VS, and BD guided the system implementation and requirements. Statistics and data processing were overseen by MG, and he also drafted the manuscript. All authors read and approved the final manuscript.

\section{Acknowledgements}

We would like to acknowledge Linda Moore and Michael Garcia from Houston Methodist for their support and feedback regarding the SmartOR system, as well as for discussions regarding patient outcomes correlation with OR efficiency.

We would also like to acknowledge the Houston Methodist OR staff for their cooperation and enthusiasm for this study.

\section{Author details}

${ }^{1}$ Department of Biology, University of Houston, 4800 Calhoun Rd, Houston TX 77004, USA. ${ }^{2}$ LASIE UMR CNRS, University La Rochelle, Avenue Michel Crepeau, 17042 La Rochelle, Cedex 1, France. ${ }^{3}$ Department of Surgery, Houston Methodist Hospital, 6550 Fannin St. Suite 1661, Houston TX 77030, USA. ${ }^{4}$ Department of Computer Science, University of Houston, 6100 Main St., Houston TX 77005, USA. ${ }^{5}$ Duke University, 102 Wannamaker Fire Ln., Wannamaker 012, 99718, Durham, North Carolina 27708, USA. 
Received: 2 January 2015 Accepted: 7 July 2015

Published online: 18 July 2015

\section{References}

1. Dexter F, Abouleish AE, Epistein RH, Whitten CW, Lubarsky DA. Use of operating room information system data to predict the impact of reducing turnover times on staffing costs. Anesth Analg. 2003;97:1119-26.

2. Strum DP, Vargas $L G, M a y J H$, Bashein G. Surgical suite utilization and capacity planning: a minimal cost analysis model. J Med Syst. 1997;21:309-22.

3. Agarwal S, Joshi A, Finin T, Yesha Y. A pervasive computing system for the operating room of the future. Mobile Networks and Applications. 2007;12:215-28.

4. Dexter F, Willemsen-Dunlap A, Lee J. Operating room managerial decision-making on the day of surgery with and without computer recommendations and status displays. Anesth Analg. 2007;105:419-29.

5. Liu CC, Chang CH, Su MC, Chu HT, Hung SH, Wong JM, et al. RFID-initiated workflow control to facilitate patient safety and utilization efficiency in operation theater. Comput Methods Prog Biomed. 2011;3:435-42.

6. Harrop JS, Styliaras JC, Ooi YC, Radcliff KE, Vaccaro AR, Wu C. Contributing factors to surgical site infections. J Am Acad Orthop Surg. 2012;20:94-101.

\section{Submit your manuscript to a SpringerOpen ${ }^{\circ}$ journal and benefit from:}

- Convenient online submission

- Rigorous peer review

- Immediate publication on acceptance

- Open access: articles freely available online

- High visibility within the field

- Retaining the copyright to your article 